Article

\title{
Strong coupling between plasmonic surface lattice resonance and photonic microcavity modes
}

\author{
Yunjie Shi ${ }^{1,2}$, Wei Liu ${ }^{2,3}$, Shidi Liu ${ }^{2,4}$, Tianyu Yang ${ }^{2,5}$, Yuming Dong ${ }^{2,5}$, Degui Sun ${ }^{1 *}$, and \\ Guangyuan $\mathrm{Li}^{2,5 * \mathbb{D}}$ \\ 1 Schools of Science, Changchun University of Science and Technology, 7089 Weixing Road, Changchun, \\ 130022, China \\ 2 CAS Key Laboratory of Human-Machine Intelligence-Synergy Systems, Shenzhen Institute of Advanced \\ Technology, Chinese Academy of Sciences, Shenzhen 518055, China \\ 3 School of Physics and Electronic Engineering, Chongqing Normal University, Chongqing 401331, P.R. China \\ 4 Wuhan Research Institute of Posts and Telecommunications, Wuhan 430070, China \\ 5 Shenzhen College of Advanced Technology, University of Chinese Academy of Sciences, Shenzhen 518055, \\ China \\ * Correspondence: sundg@cust.edu.cn; gy.li@siat.ac.cn
}

\begin{abstract}
We report the strong coupling between plasmonic surface lattice resonances (SLRs) and photonic Fabry-Pérot (F-P) resonances in a microcavity embedded with two-dimensional periodic array of metal-insulator-metal nanopillars. For such a plasmonic-photonic system, we show that the SLR can be strongly coupled to the F-P resonances of both the odd- and even orders, and that the splitting energy reaches as high as $138 \mathrm{meV}$ in the visible regime. We expect that this work will provide a new scheme for strong coupling between plasmonic and photonic modes.
\end{abstract}

Keywords: Strong coupling; plasmonic-photonic coupling system; surface lattice resonance; Fabry-Pérot resonance.

\section{Introduction}

Strong coupling between photonic cavities and plasmons have been of increasing interest [1-6] since the first demonstration between localized plasmons in nanowire pairs and photonic Fabry-Pérot (F-P) microcavity modes by Ameling and Giessen in 2010 [7]. By combining plasmonic nanostructures and photonic microcavities, it has been demonstrated that the sensing properties of localized plasmon sensors can be enhanced [8-10], that up to two-fold enhancement increase can be achieved compared to that without using the cavity [11], that large Rabi splitting can be obtained by embedding nanoparticle-WS $\mathrm{W}_{2}$ heterostructure in an optical microcavity $[12,13]$, and that both peak splitting and peak locking behaviors can be observed [14].

A key figure of merit for these strong coupling effects is the splitting energy. For example, Ameling and Giessen [7] showed that the splitting energy for the electric mode can be as large as $358 \mathrm{meV}$ in the near-infrared regime, corresponding to $36 \%$ of the resonant energy. Quite recently, Baranov et al. [15] further demonstrated ultrastrong coupling between nanoparticle plasmons and cavity photons at ambient conditions, and achieved giant Rabi splitting energy of $1 \mathrm{eV}$ and extremely large splitting/resonance energy ratio of $120 \%$.

Besides the strong coupling between localized plasmons and photonic F-P cavity modes [1,7], the strong coupling between other types of plasmons and other photonic modes has also been extensively investigated. Ameling et al. [16] demonstrated that both the localized and propagating surface plasmons can couple strongly with photonic microcavity modes, and the splitting energies were calculated to be $141 \mathrm{meV}$ and $224 \mathrm{meV}$, corresponding to $\sim 10 \%$ of the resonant energy in the 
near-infrared regime. Chen et al. [17] investigated the strong coupling between propagating surface plasmons and the F-P cavity and observed a large Rabi-analogue splitting energy of $148 \mathrm{meV}$ in the visible regime. Wang et al. [18] adopted another configuration and obtained $31.5 \mathrm{meV}$ around the resonant wavelength of $747 \mathrm{~nm}$. Similarly, Alrasheed and Fabrizio [11] also obtained a Rabi splitting of $76 \mathrm{meV}$ in the visible. Additionally, the strong coupling between plasmonic gap modes and photonic lattice modes was also reported by Lin et al. [19] and Saito et al. [20], respectively, and a Rabi splitting of $\sim 110 \mathrm{meV}$ was obtained [19]. Chen et al. [21] investigated the strong coupling between magnetic plasmons and photonic F-P cavity modes and obtained a Rabi-type splitting of $78 \mathrm{meV}$ around the resonant wavelength of $772 \mathrm{~nm}$.

Recently, lattice plasmons, which are usually referred to as plasmonic surface lattice resonances (SLRs), have emerged as an excellent platform for light-matter interactions on the nanoscale because of the attractive merits such as strong field enhancement extended over large volumes, and large wavelength tunability [22-24]. Taking advantage of the strong and delocalized electromagnetic fields associated with the SLRs, great progress has been achieved in the strong coupling between SLRs and different materials [6] such as organic molecules [25,26], transition metal dichalcogenides [27], and quantum dots [28]. However, the strong coupling between SLRs and photonic F-P cavity modes has not been explored yet.

In this work, we report, for the first time, the strong coupling between SLRs and photonic F-P microcavity modes by placing a thin gold film on top of a two-dimensional (2D) array of metal-insulator-metal (MIM) nanopillars. The nanopillars stand on a silica $\left(\mathrm{SiO}_{2}\right)$ substrate and are surrounded by air. As we have demonstrated previously, the SLR supported by the nanopillar array prefers such an asymmetric dielectric environment [29]. Here we will show that, the SLR can strongly couple with the photonic modes of both odd and even orders supported by the F-P microcavity formed by the thin gold film and the air $/ \mathrm{SiO}_{2}$ interface, and splitting energies as high as $138.4 \mathrm{meV}$ can be obtained in the visible, corresponding to $8.6 \%$ of the resonant energy.

\section{Structure design and numerical setups}

Figure 1(a) illustrates the strongly coupled plasmonic-photonic system under study, which is composed of a 2D periodic array of MIM nanopillars standing on a silica substrate and a thin gold film of thickness $20 \mathrm{~nm}$ on the top. The distance between the bottom surface of the gold film and the substrate top surface is $L$. The nanopillars with square-shaped cross section have side length of $w$ and period of $\Lambda$ in both the $x$ and $y$ directions. The thicknesses of the top and bottom gold nanoridges are $h_{\mathrm{t}}$ and $h_{\mathrm{b}}$, respectively, and the thickness of the central insulating silica layer is $h_{\mathrm{I}}$. The structure is normally illuminated by a plane wave with electric field of $x$ polarization and of unitary amplitude.

The reflectance spectra, as well as the near-field distributions of the plasmonic-photonic coupling system were simulated using an in-house package for fully vectorial rigorous coupled-wave analysis (RCWA) developed following [30-32]. As references, we also calculated the optical properties of the reference F-P cavity formed by the gold film and the air $/ \mathrm{SiO}_{2}$ interface, and the $2 \mathrm{D}$ array of MIM nanopillars, which are illustrated by Figs. 1(b)(c), respectively. In all the simulations, we adopted retained orders of $41 \times 41$, which were shown to be large enough to reach the convergence regime. Wavelength-dependent permittivities of gold are taken from Johnson \& Christy [33], and the refractive indices of the substrate and the central silica are set to be $n_{\text {sub }}=n_{\mathrm{I}}=1.45$. We set $h_{\mathrm{t}}=h_{\mathrm{b}}=140 \mathrm{~nm}$, $h_{\mathrm{I}}=260 \mathrm{~nm}, w=200 \mathrm{~nm}$, and $\Lambda=510 \mathrm{~nm}$.

\section{Results and Discussion}

Figure 1(d) depicts the simulated reflectance spectra of the proposed plasmonic-photonic coupling system and the reference F-P cavity with $h=840 \mathrm{~nm}$, and the MIM nanopillar array. Results show that for the MIM nanopillar array in asymmetric dielectric environment, the reflectance spectra exhibits Fano-type asymmetric peak-and-dip spectral feature. A narrow dip is centered at $\lambda_{\mathrm{SLR}}=766.8 \mathrm{~nm}$ and has full-width-half-maximum of $20.6 \mathrm{~nm}$. Thus the quality factor is estimated to be $Q=37$, which 


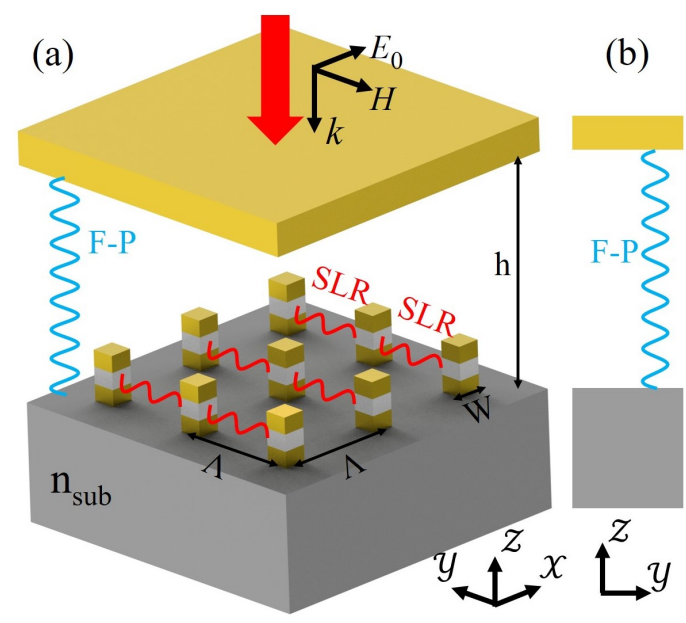

(c)

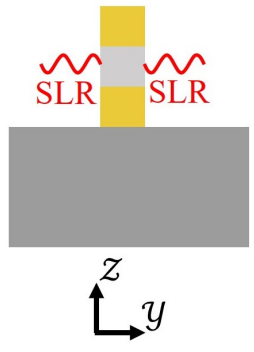

(d)

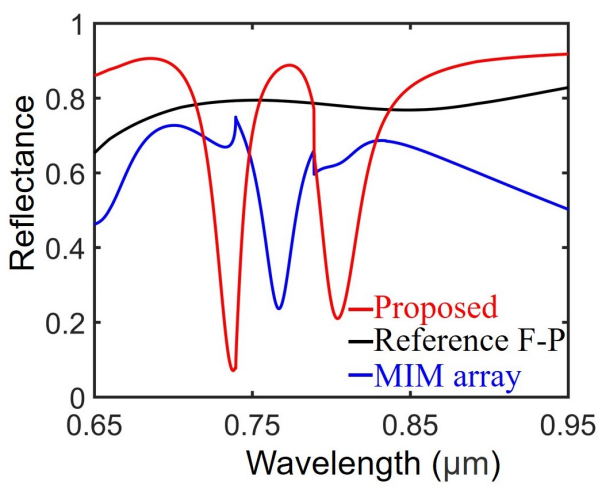

Figure 1. (a) Schematic of the strong coupling between the SLR and the F-P resonance in the proposed structure composed of a thin metal film and a 2D array of MIM nanopillars on a quartz substrate. (b)(c) schematics of the side views of (b) the reference F-P cavity that supports photonic resonances and (c) the MIM nanopillar array that supports SLR. (d) Simulated reflectance spectra of the proposed plasmonic-photonic system (red curve), the reference F-P cavity (black curve) and the MIM nanopillar array (blue curve). The calculations were performed with $h=840 \mathrm{~nm}$.

is about twice of that of typical localized plasmons $(Q \sim 20$ in the visible). According to our previous study [29], this dip should corresponds to a SLR, which will be further confirmed later. For the reference F-P cavity, photonic resonance can be observed at $\lambda_{\mathrm{F}-\mathrm{P}}=844.8 \mathrm{~nm}$. For the plasmonic-photonic coupling system, we observe that the SLR (blue curve) is split into two modes (red curve) that are resonant at $\lambda_{\mathrm{L}}=737.8 \mathrm{~nm}$ and $\lambda_{\mathrm{R}}=804 \mathrm{~nm}$. The Rabi-analogue splitting energy reaches $138 \mathrm{meV}$, which is about $8.6 \%$ of the resonance energy. This confirms that the proposed plasmonic-photonic system is in the strong coupling regime. This splitting energy is much larger than those of many plasmonic-photonic strong coupling systems operating in the visible $[11,18,19,21,34]$.

In order to gain a deeper insight concerning the nature of the split modes, in Fig. 2 we plot the near-field electric field distributions $\left(|E|^{2}\right)$ at these four resonance wavelengths. Fig. 2(a) shows that at $\lambda_{\mathrm{SLR}}=766.8 \mathrm{~nm}$, in-plane dipolar oscillations and out-of-plane quadrupolar oscillations are excited in the top and bottom gold ridges, respectively. The electric field is greatly enhanced around the gold corners while extending over large volumes. These near-field characteristics are consistent with our previous study [29], further validating the excitation of SLR. Fig. 2(b) shows the electric field distributions of the reference F-P cavity at $\lambda_{\mathrm{F}-\mathrm{P}}=844.8 \mathrm{~nm}$. It is evident that the second-order F-P resonance is excited. The electric field within the cavity is relatively weak since the reflectance of the air $/ \mathrm{SiO}_{2}$ interface is very small $(\sim 5 \%)$. This corresponds to weak F-P resonance, as evidenced by the small modulation depth in the reflectance spectra, as shown by the black curve in Fig. 1(d).

For the proposed plasmonic-photonic coupling system, Figs. 2(c)(d) show the hybridization of the SLR in the MIM nanopillar array and the second-order photonic F-P cavity mode into a symmetric mode at $\lambda_{\mathrm{L}}=737.8 \mathrm{~nm}$ and an antisymmetric mode at $\lambda_{\mathrm{R}}=804 \mathrm{~nm}$. For the former, the electric fields around the top gold ridge point in the same direction as the bottom electric fields of the second-order F-P mode. Hence the electric fields around the top gold ridge are greatly enhanced with large volume extension. However, for the latter, the electric fields around the top gold ridge point in the opposite direction, resulting in cancellation of the dipolar oscillation in the top gold ridge. This results in greatly enhanced electric fields around the bottom gold ridge with large volume extension.

We now investigate the effects of the F-P cavity thickness on the plasmonic-photonic strong coupling system. Fig. 3(a) depicts the photonic resonant modes of the reference F-P cavity with thicknesses from $h=0.64 \mu \mathrm{m}$ to $h=3.04 \mu \mathrm{m}$. When the MIM nanopillar array is placed into the F-P resonator, the interactions between the photonic F-P resonant modes and the SLR result in anticrossings 

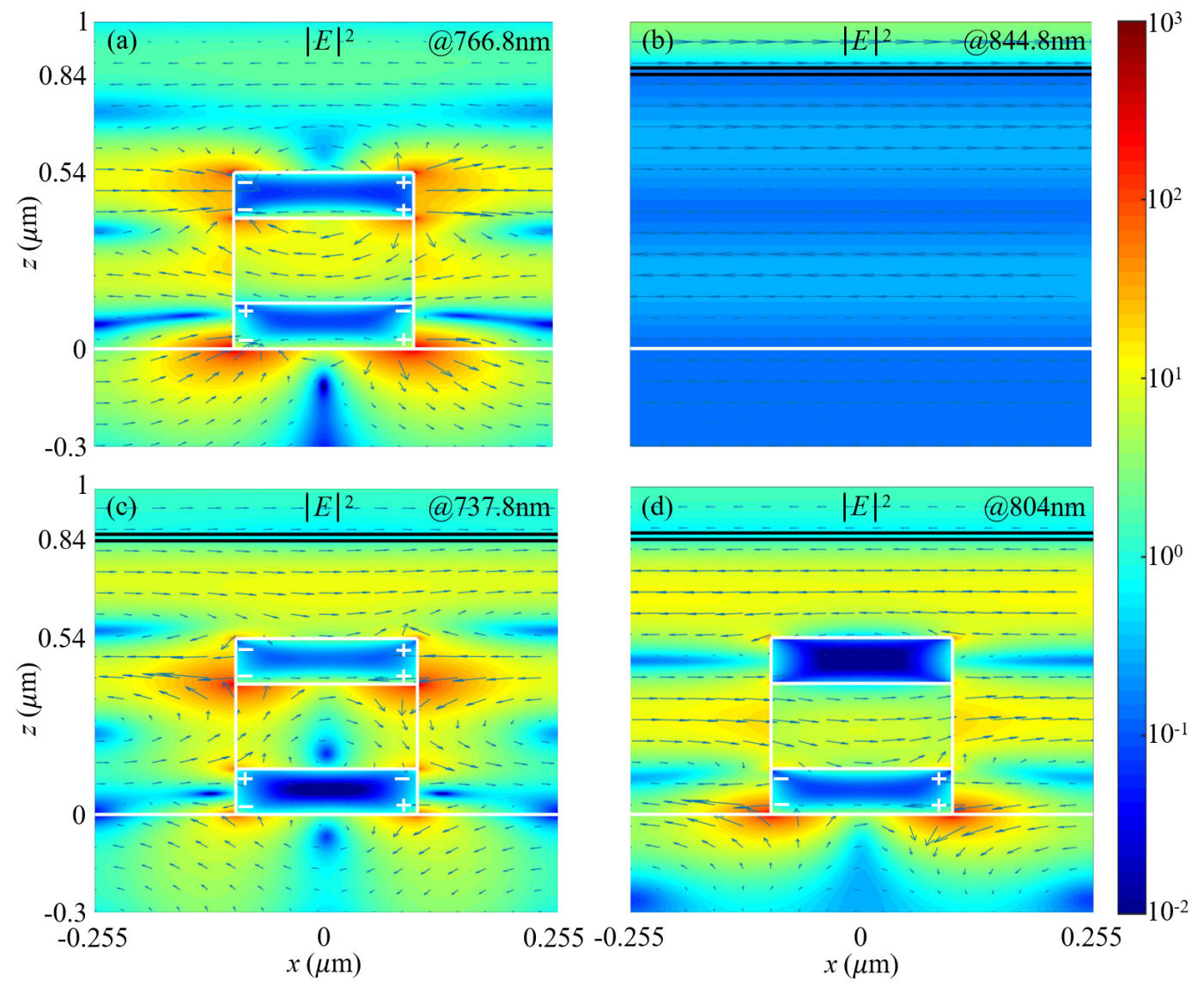

Figure 2. Simulated electric field distributions $|E|^{2}$ of (a) the MIM nanopillar array at $\lambda_{\mathrm{SLR}}=766.8 \mathrm{~nm}$, (b) the reference F-P cavity at $\lambda_{\mathrm{F}-\mathrm{P}}=844.8 \mathrm{~nm},(\mathrm{c})(\mathrm{d})$ the proposed structure of (c) $\lambda_{\mathrm{L}}=737.8 \mathrm{~nm}$ and $\lambda_{\mathrm{R}}=804 \mathrm{~nm}$. The MIM nanopillar array is outlined by white lines and the thin metal film on the top is outlined by black lines. "+" and "-" in $(\mathrm{a})(\mathrm{c})(\mathrm{d})$ indicate charge distributions.
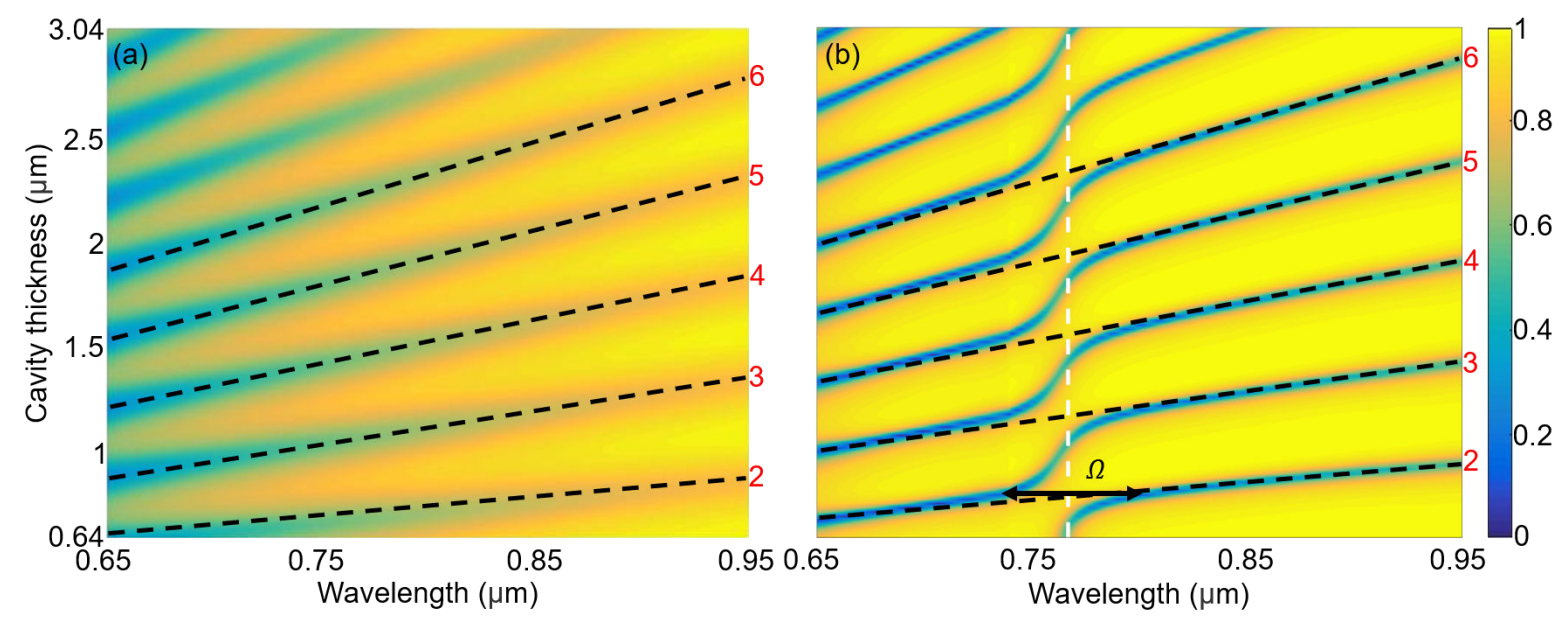

Figure 3. Simulated reflectance spectra of (a) reference F-P cavity and (b) proposed plasmonic-photonic coupling system as functions of the cavity length. The vertical white dashed line in (b) indicates SLR wavelength of the MIM nanopillar array. Anti-crossings are observable around every intersection of the F-P modes and the SLR mode. 
of these modes. Strikingly, all orders of photonic F-P resonances can interact with the SLR supported by the MIM nanopillar array. This behavior is distinct from that of the strong coupling between localized plasmons and photonic F-P modes, for which the former can interact with every second photonic F-P modes (odd or even, depending on the characteristics of the localized plasmons) $[1,7,15]$. As the F-P resonance order increases, the splitting energy decreases significantly. This is similar to the strong coupling between localized plasmons and photonic F-P modes.

Comparing Figs. 3(a) and (b), we notice that there are deviations on the photonic F-P resonances between the reference F-P cavity and the proposed plasmonic-photonic coupling system, in terms of not only the resonance wavelengths but also the modulation depths. These deviations arise because the metal ridges provide additionally reflectance besides the air $/ \mathrm{SiO}_{2}$ interface of the reference F-P cavity. This also explains the mismatch of the reference F-P resonance wavelength and the SLR wavelength of the MIM nanopillar array in Fig. 1(d).
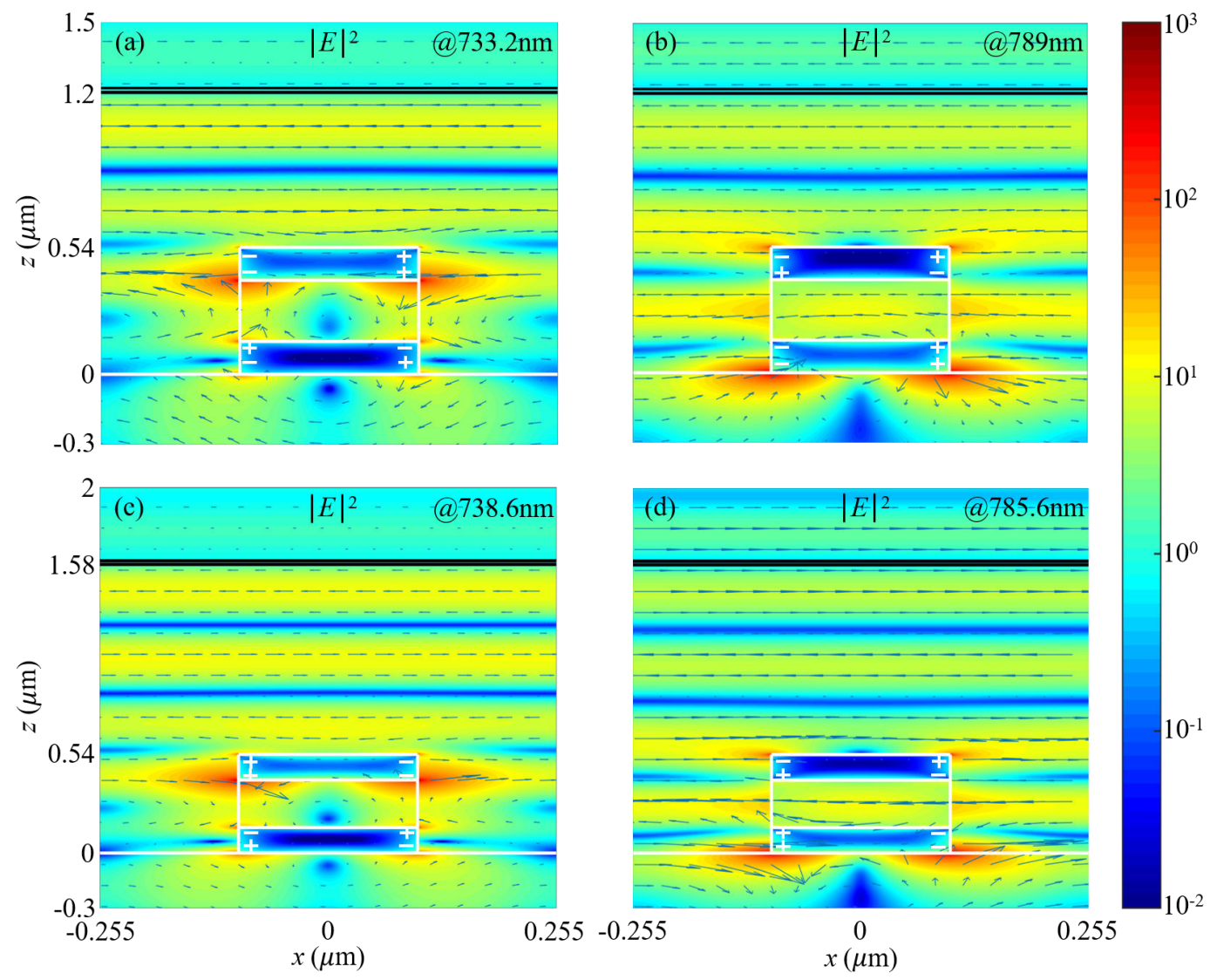

Figure 4. Similar to Fig. 2(c)(d) but at (a) $\lambda_{\mathrm{L}}=733.2 \mathrm{~nm}$ and (b) $\lambda_{\mathrm{R}}=789 \mathrm{~nm}$ for $h=1.2 \mu \mathrm{m}$ supporting the third-order photonic F-P resonance, and (c) $\lambda_{\mathrm{L}}=738.6 \mathrm{~nm}$ and (d) $\lambda_{\mathrm{R}}=785.6 \mathrm{~nm}$ for $h=1.58 \mu \mathrm{m}$, with which the reference F-P cavity supports the fourth-order resonance.

Figure 4 depict the near-field electric fields due to the hybridization of the SLR and the third- or fourth-order F-P resonance. It is clear that in general, the electric field distributions of the symmetric modes at low wavelengths and of the antisymmetric modes at high wavelengths are similar to their counterparts in Fig. 2(c)(d). To be more specifically, the overall field distributions follow those of the photonic F-P modes, and the electric fields are greatly enhanced over large volumes around the top or the bottom metal ridge for the symmetric or antisymmetric modes.

\section{Conclusion}

In conclusion, we have numerically observed strong coupling between SLR and photonic F-P resonances of both even and odd orders in the proposed plasmonic-photonic coupling system. We 
have found that the Rabi splitting energy reaches $138 \mathrm{meV}$, which is about $8.6 \%$ of the resonance energy in the visible regime. For the strong coupling between the SLR and photonic F-P resonances of different order, we have found that the electric fields are greatly enhanced with large volume extension around the top metal ridge for the symmetric modes at low wavelengths, or around the bottom metal ridge for the antisymmetric modes at high wavelengths. We expect that the proposed plasmonic-photonic strong coupling system will provide a new scheme for strong coupling between plasmonic and photonic modes, and find potential applications in manipulation of the near field distributions and sensing.

Author Contributions: conceptualization, G.L.; methodology, Y.S.; software, G.L.; validation, Y.S.; formal analysis, Y.S., W.L., S.L., and G.L.; investigation, Y.S.; resources, Y.D. and G.L.; data curation, Y.S. and W.L.; writing-original draft preparation, Y.S.; writing-review and editing, T.Y., D.S., Y.D. and G.L.; visualization, Y.S.; supervision, D.S., Y.D. and G.L.; project administration, G.L.; funding acquisition, Y.D. and G.L..

Funding: This research was funded by the State Key Laboratory of Advanced Optical Communication Systems Networks, China (2020GZKF004) and Shenzhen Research Foundation (JCYJ20170413152328742).

Conflicts of Interest: The authors declare no conflict of interest.

\section{References}

1. Ameling, R.; Giessen, H. Microcavity plasmonics: strong coupling of photonic cavities and plasmons. Laser Photonics Rev. 2013, 7, 141-169.

2. Baranov, D.G.; land Jorge Cuadra, M.W.; Antosiewicz, T.J.; .; Shegai, T. Novel Nanostructures and Materials for Strong Light-Matter Interactions. ACS Photonics 2018, 5, 24-42.

3. Dovzhenko, D.S.; Ryabchuk, S.V.; Rakovich, Y.P.; Nabiev, I.R. Light-matter interaction in the strong coupling regime: configurations, conditions, and applications. Nanoscale 2018, 10, 3589-3605.

4. Hertzog, M.; Wang, M.; Mony, J.; B orjesson, K. Strong light-matter interactions: a new direction within chemistry. Chem. Soc. Rev. 2019, 48, 937-961.

5. Yu, X.; Yuan, Y.; Xu, J.; Yong, K.T.; Qu, J.; Song, J. Strong Coupling in Microcavity Structures: Principle, Design, and Practical Application. Laser Photonics Rev. 2019, 13, 1800219.

6. Ramezani, M.; Berghuis, M.; Rivas, J.G. Strong light-matter coupling and exciton-polariton condensation in lattices of plasmonic nanoparticles [Invited]. J. Opt. Soc. Am. B 2019, 36, E88-E103.

7. Ameling, R.; Giessen, H. Cavity Plasmonics: Large Normal Mode Splitting of Electric and Magnetic Particle Plasmons Induced by a Photonic Microcavity. Nano Lett. 2010, 10, 4394-4398.

8. Ameling, R.; Langguth, L.; Hentschel, M.; Mesch, M.; Braun, P.V.; Giessen, H. Cavity-enhanced localized plasmon resonance sensing. Appl. Phys. Lett. 2010, 97, 253116.

9. Schmidt, M.A.; Lei, D.Y.; Wondraczek, L.; Nazabal, V.; Maier, S.A. Hybrid nanoparticle-microcavity-based plasmonic nanosensors with improved detection resolution and extended remote-sensing ability. Nat. Commun. 2012, 3, 1108.

10. Chen, J.; Nie, H.; Peng, C.; Qi, S.; Tang, C.; Zhang, Y.; Wang, L.; Park, G.S. Enhancing the Magnetic Plasmon Resonance of Three-Dimensional Optical Metamaterials via Strong Coupling for High-Sensitivity Sensing. J. Lightwave Technol. 2018, 36, 3481-3485.

11. Alrasheed, S.; Fabrizio, E.D. Effect of Surface Plasmon Coupling to Optical Cavity Modes on the Field Enhancement and Spectral Response of Dimer-Based sensors. Sci. Rep. 2017, 7, 10524.

12. Li, B.; Zu, S.; Zhang, Z.; Zheng, L.; Jiang, Q.; Du, B.; Luo, Y.; Gong, Y.; Zhang, Y.; Lin, F.; Shen, B.; Zhu, X.; Ajayan, P.M.; Fang, Z. Large Rabi splitting obtained in Ag-WS2 strong-coupling heterostructure with optical microcavity at room temperature. Opto-Electronic Advances 2019, 2, 190008.

13. Bisht, A.; Cuadra, J.; Wers all, M.; Canales, A.; Antosiewicz, T.J.; Shegai, T. Collective Strong Light-Matter Coupling in Hierarchical Microcavity-Plasmon-Exciton Systems. Nano Lett. 2019, 19, 189-196.

14. Li, P.; Ji, L.; Gao, N.; Wang, H.; Ge, S.; Huang, K.; Kang, J.; Yu, E.T. Peak splitting and locking behavior arising from Fano interference between localized surface plasmons and cavity modes. Phys. Rev. B 2019, 99, 125420.

15. Baranov, D.G.; Munkhbat, B.; Zhukova, E.; Bisht, A.; Canales, A.; Rousseaux, B.; Johansson, G.; Antosiewicz, T.J.; Shegai, T. Ultrastrong coupling between nanoparticle plasmons and cavity photons at ambient conditions. Nat. Commun. 2020, 11, 2715. 
16. Ameling, R.; Dregely, D.; Giessen, H. Strong coupling of localized and surface plasmons to microcavity modes. Opt. Lett. 2011, 36, 2218-2220.

17. Chen, S.; Li, G.; Lei, D.; Cheah, K.W. Efficient energy exchange between plasmon and cavity modes via Rabi-analogue splitting in a hybrid plasmonic nanocavity. Nanoscale 2013, 5, 9129-9133.

18. Wang, Y.; Sun, C.; Gan, F.; Li, H.; Gong, Q.; Chen, J. Sharp phase variations from the plasmon mode causing the Rabi-analogue splitting. Nanophotonics 2017, 6, 1101-1107.

19. Lin, Q.Y.; Li, Z.; Brown, K.A.; O’Brien, M.N.; Ross, M.B.; Zhou, Y.; Butun, S.; Chen, P.C.; Schatz, G.C.; Dravid, V.P.; Aydin, K.; Mirkin, C.A. Strong Coupling between Plasmonic Gap Modes and Photonic Lattice Modes in DNA-Assembled Gold Nanocube Arrays. Nano Lett. 2015, 15, 4699-4703.

20. Saito, H.; Yoshimoto, D.; Lourenco-Martins, H.; Yamamoto, N.; Sannomiya, T. Hybridization of Gap Modes and Lattice Modes in a Plasmonic Resonator Array with a Metal-Insulator-Metal Structure. ACS Photonics 2019, 6, 2618-2625.

21. Chen, J.; Zhang, T.; Tang, C.; Mao, P.; Liu, Y.; Yu, Y.; Liu, Z. Optical Magnetic Field Enhancement via Coupling Magnetic Plasmons to Optical Cavity Modes. IEEE Photon. Technol. Lett. 2016, 28, 1529-1532.

22. Wang, W.; Ramezani, M.; Väkeväinen, A.I.; Törmä, P.; Rivas, J.G.; Odom, T.W. The Rich Photonic World of Plasmonic Nanoparticle Arrays. Mater. Today 2018, 21, 303-314.

23. Kravets, V.G.; Kabashin, A.V.; Barnes, W.L.; Grigorenko, A.N. Plasmonic Surface Lattice Resonances: A Review of Properties and Applications. Chem. Rev. 2018, 118, 5912-5951.

24. Wang, D.; Guan, J.; Hu, J.; Bourgeois, M.R.; Odom, T.W. Manipulating Light-Matter Interactions in Plasmonic Nanoparticle Lattices. Acc. Chem Res. 2019, 52, 2997-3007.

25. V akev ainen, A.I.; Moerland, R.J.; Rekola, H.T.; Eskelinen, A.P.; Martikainen, J.P.; Kim, D.H.; T orm a, P. Plasmonic Surface Lattice Resonances at the Strong Coupling Regime. Nano Lett. 2014, 14, 1721-1727.

26. Shi, L.; Hakala, T.K.; Rekola, H.T.; Martikainen, J.P.; Moerland, R.J.; T orm a, P. Spatial Coherence Properties of Organic Molecules Coupled to Plasmonic Surface Lattice Resonances in the Weak and Strong Coupling Regimes. Phys. Rev. Lett. 2014, 112, 153002.

27. Wang, S.; Le-Van, Q.; Vaianella, F.; Maes, B.; Barker, S.E.; Godiksen, R.H.; Curto, A.G.; Rivas, J.G. Limits to Strong Coupling of Excitons in Multilayer WS2 with Collective Plasmonic Resonances. ACS Photonics 2019, 6, 286-293.

28. Yadav, R.K.; Bourgeois, M.R.; Cherqui, C.; Juarez, X.G.; Wang, W.; Odom, T.W.; Schatz, G.C.; Basu, J.K. Room Temperature Weak-to-Strong Coupling and the Emergence of Collective Emission from Quantum Dots Coupled to Plasmonic Arrays. ACS Nano 2020, 14, 7347-7357.

29. Yang, X.; Xiao, G.; Lu, Y.; Li, G. Narrow plasmonic surface lattice resonances with preference to asymmetric dielectric environment. Opt. Express 2019, 27, 25384-25394.

30. Moharam, M.G.; Pommet, D.A.; Grann, E.B.; Gaylord, T.K. Stable Implementation of the Rigorous Coupled-Wave Analysis for Surface-Relief Gratings: Enhanced Transmittance Matrix Approach. J. Opt. Soc. Am. A 1995, 12, 1077-1086.

31. Lalanne, P. Improved formulation of the coupled-wave method for two-dimensional gratings. J. Opt. Soc. Am. A 1997, 14, 1592-1598.

32. David, A.; Benisty, H. Fast factorization rule and plane-wave expansion method for two-dimensional photonic crystals with arbitrary hole-shape. Phys. Rev. B: Condens. Matter Mater. 2006, 73, 075107.

33. Johnson, P.B.; Christy, R.W. Optical Constants of the Noble Metals. Phys. Rev. B 1972, 6, 4370-4379.

34. Alast, F.H.; Li, G.; Cheah, K.W. Rabi-like splitting from large area plasmonic microcavity. AIP Adv. 2017, 7, 085201. 